\title{
HR 8257: A THREE-DIMENSIONAL ORBIT AND BASIC PROPERTIES
}

\author{
Francis C. Fekel ${ }^{1,5}$, Andrew F. Boden ${ }^{2}$, Jocelyn Tomkin ${ }^{3}$, And Guillermo Torres ${ }^{4}$ \\ ${ }^{1}$ Center of Excellence in Information Systems, Tennessee State University, Nashville, TN 37209, USA; fekel@evans.tsuniv.edu \\ 2 Division of Physics, Math, and Astronomy, California Institute of Technology, Pasadena, CA 91125, USA; bode@ astro.caltech.edu \\ ${ }^{3}$ Department of Astronomy and McDonald Observatory, University of Texas, Austin, TX 78712, USA; jt@alexis.as.utexas.edu \\ ${ }^{4}$ Harvard-Smithsonian Center for Astrophysics, Cambridge, MA 02138, USA; gtorres@cfa.harvard.edu \\ Received 2008 December 8; accepted 2009 January 28; published 2009 April 7
}

\begin{abstract}
We have used interferometric and spectroscopic observations of HR 8257 to determine a three-dimensional orbit of the system. The orbit has a period of 12.21345 days and an eccentricity of 0.2895 . The masses of the F0 and F2 dwarf components are 1.56 and $1.38 M_{\odot}$, respectively, with fractional errors of $1.4 \%$. Our orbital parallax of $13.632 \pm 0.095$ mas, corresponding to a distance of $73.4 \pm 0.6 \mathrm{pc}$, differs from the Hipparcos result by just $2 \%$ and has a significantly smaller uncertainty. From our spectroscopic observations and spectral energy distribution modeling we determine the component effective temperatures and luminosities to be $T_{\text {eff }}(\mathrm{A})=7030 \pm 200 \mathrm{~K}$ and $T_{\text {eff }}(\mathrm{B})=6560 \pm 200 \mathrm{~K}$ and $L_{A}=9.4 \pm 0.3 L_{\odot}$ and $L_{B}=4.7 \pm 0.2 L_{\odot}$. The primary rotates pseudosynchronously, while the secondary is not far from its pseudosynchronous rotational velocity. Although both early-F stars are slowly rotating, neither component of this close binary is an Am star. A comparison with evolutionary tracks indicates that the stars are slightly metal poor, and although the components have evolved away from the zero-age main sequence, they are both still dwarfs.
\end{abstract}

Key words: binaries: spectroscopic - stars: fundamental parameters - stars: individual (HR 8257)

Online-only material: machine-readable table

\section{INTRODUCTION}

HR 8257 (HD $205539=$ HIP 106595; $\alpha=21^{\mathrm{h}} 35^{\mathrm{m}} 19.12$, $\delta=28^{\circ} 11^{\prime} 51^{\prime \prime} .4$ [J2000.0], $V=6.25 \mathrm{mag}$ ) has been known as a double-lined spectroscopic binary for more than $80 \mathrm{yr}$. From velocities obtained in 1923-1925, Harper (1925) computed an initial orbit and found a period of 12.21 days and an eccentricity of 0.32 . Using five additional observations, acquired nearly a decade later, Harper (1935) revised the period to 12.216 days. The combined spectrum of the stars has been classified as F2 IV,V by W. Morgan (Abt \& Bidelman 1969), F0 IV (Cowley \& Fraquelli 1974), and F2 IVp (Abt \& Morrell 1995). Petrie (1950) determined a visual magnitude difference of 0.67 for the components. McAlister et al. (1987) examined 672 bright stars for close visual companions. As a result, they announced the discovery of 52 newly resolved binaries, including HR 8257, which was given the discovery designation of CHARA 103 . The separation of 0 '. 18 found for the binary suggested an orbital period of roughly 100 yr. However, McAlister et al. (1993) reported that HR 8257 was one of 18 binaries for which five to 10 follow-up observations, obtained over the next several years, did not confirm the earlier announced duplicity, and so the claim was withdrawn.

HR 8257 is one of over 40 spectroscopic binary stars with previously determined orbits that was chosen for reobservation in the ongoing project of Fekel \& Tomkin (2004). First results were reported by Tomkin \& Fekel (2006). HR 8257 was also on the spectroscopic observing program of $\mathrm{G}$. Torres at the Harvard-Smithsonian Center for Astrophysics (CfA), as well as the Palomar Testbed Interferometer (PTI) binary star program of A. Boden. Learning of our mutual interest in this system, the

\footnotetext{
5 Visiting Astronomer, Kitt Peak National Observatory, National Optical Astronomy Observatory, operated by the Association of Universities for Research in Astronomy, Inc. under cooperative agreement with the National Science Foundation.
}

extensive new observations have been combined to produce a greatly improved description of the system.

\section{OBSERVATIONS AND REDUCTIONS}

\subsection{Interferometric}

The PTI is a long-baseline $H-(1.6 \mu \mathrm{m})$ and $K$-band $(2.2 \mu \mathrm{m})$ interferometer that is located at the Palomar Observatory, and described in detail elsewhere (Colavita et al. 1999). HR 8257 was added to its observing list in 2000, and the PTI was used to make the interferometric measurements presented here. Like previous orbital determinations that used PTI data (Boden et al. 2005, 2006), the interferometric observable for these measurements is the fringe contrast or visibility (squared), $V^{2}$, of an observed brightness distribution on the sky. The analysis of such data for binary orbital estimation is discussed in detail in previous work (e.g., Boden et al. 2000) and elsewhere (e.g., Hummel et al. 1998) and is not repeated here.

Our $K$-band observations of HR 8257 and the objects in our calibrator list were obtained with the PTI on 58 nights between 2000 June 25 and 2005 October 14, a data set spanning roughly $5.3 \mathrm{yr}$ and 159 orbital periods. On each night HR 8257 and the calibration objects were typically observed multiple times, and each observation or scan took approximately $130 \mathrm{~s}$. For each scan we computed a mean $V^{2}$ value from the scan data and the error in the $V^{2}$ estimate from the rms internal scatter (Colavita 1999; Boden et al. 1998). HR 8257 was observed in combination with one or more calibration sources within $\sim 10^{\circ}$ on the sky. Here we have used three giant stars as calibration objects, HD 199763, HD 206646, and HD 211432, and list their relevant physical parameters in Table 1. Calibrating our interferometric data with these objects results in $350 \mathrm{~K}$-band visibility scans on HR 8257. The calibrated $V^{2}$ data on HR 8257 are summarized in Table 2. 
Table 1

HR 8257 PTI $V^{2}$ Calibration Objects

\begin{tabular}{lcccc}
\hline \hline Star & $\begin{array}{c}\text { Spectral } \\
\text { Type }\end{array}$ & $\begin{array}{c}\text { Star } \\
\text { Magnitude } \\
(\mathrm{mag})\end{array}$ & $\begin{array}{c}\text { Separation } \\
\text { from HR 8257 } \\
(\mathrm{deg})\end{array}$ & $\begin{array}{c}\text { Adopted Model } \\
\text { Diameter } \\
(\mathrm{mas})\end{array}$ \\
\hline HD 199763 & G9 III & $6.5 \mathrm{~V} / 4.3 \mathrm{~K}$ & 8.4 & $0.679 \pm 0.060$ \\
HD 206646 & K1 III & $7.0 \mathrm{~V} / 4.5 \mathrm{~K}$ & 5.2 & $0.622 \pm 0.070$ \\
HD 211432 & G9 III & $6.4 \mathrm{~V} / 4.1 \mathrm{~K}$ & 9.1 & $0.708 \pm 0.050$ \\
\hline
\end{tabular}

Notes. The relevant parameters for our two calibration objects are summarized. The apparent diameter values are determined from SED modeling, based on archival broad-band photometry, spectral energy distribution templates from Pickles (1998), and visibility measurements with PTI

\subsection{Spectroscopic}

From 2000 July to 2006 December we collected 14 observations with nearly identical echelle spectrographs mounted at the Cassegrain foci of the $1.5 \mathrm{~m}$ Wyeth reflector at the Oak Ridge Observatory, situated in Harvard, Massachusetts, and the $1.5 \mathrm{~m}$ Tillinghast reflector at the F. L. Whipple Observatory on Mount Hopkins, Arizona. The resolving power of these instruments is about 35,000 . A single order, centered at $5188.5 \AA$ near the Mg I $b$ triplet and spanning $45 \AA$, was recorded with a photoncounting Reticon detector. The signal-to-noise ratios of these Center for Astrophysics (CfA) spectra range from about 30 to 80 per resolution element.

From 2002 October to 2006 November we obtained 19 high-resolution spectrograms of HR 8257 with the McDonald Observatory $2.1 \mathrm{~m}$ telescope, the Sandiford Cassegrain echelle spectrograph (McCarthy et al. 1993), and a Reticon CCD with $27 \mu \mathrm{m}^{2}$ pixels arranged in a $1200 \times 400$ pixel format. The spectrograms cover the wavelength range 5700-7000 and have a resolving power of 49,000 . The collapsed onedimensional stellar spectra have a representative signal-to-noise ratio of $\sim 300$. Each stellar observation was followed by an observation of a thorium-argon comparison spectrum.

We acquired an additional 11 spectrograms from 2004 June to 2006 September with the Kitt Peak National Observatory (KPNO) 0.9 m coudé feed telescope, coudé spectrograph, and a TI CCD detector. All those spectrograms are centered in the red at $6430 \AA$, cover a wavelength range of $84 \AA$, and have a resolving power of over 30,000 , corresponding to a two-pixel resolution of $0.21 \AA$. Those spectra have signal-to-noise ratios of $\sim 200$.

Finally we obtained a fourth spectroscopic data set from 1982 July to 1993 June. During that period, 25 observations of HR 8257 were made with the Coravel radial velocity spectrometer (Baranne et al. 1979) and the Swiss $1 \mathrm{~m}$ telescope at the Haute-Provence Observatory (HPO).

We computed the component velocities from the CfA observations using the TODCOR two-dimensional cross-correlation technique (Zucker \& Mazeh 1994). Synthetic templates for each star were based on Kurucz model atmospheres. The optimal parameters for the templates, primarily effective temperature and rotational velocity (see Section 4), were determined by seeking the best match to the observed spectra as judged from the peak cross-correlation value averaged over all exposures.

The CfA radial velocities were tested for systematics by performing numerical simulations following Torres et al. (2002), and small adjustments, typically less than $0.5 \mathrm{~km} \mathrm{~s}^{-1}$, were applied to the raw velocities as corrections. The stablility and zero point of the velocity system were monitored by means of exposures of the dusk and dawn sky, and small systematic run-to-run corrections were applied in the manner described by Latham (1992). The final radial velocities are presented in Table 3 .

To determine the McDonald velocities, the wavelengths of well defined primary and secondary lines were measured by fitting Gaussian profiles with the SPLOT routine of IRAF, and the wavelength differences between the measured wavelengths and rest wavelengths (Moore et al. 1966) were computed. The Doppler formula converted those wavelength differences into the topocentric radial velocities for the lines. Telluric $\mathrm{O}_{2}$ lines near $6300 \AA$ ( $\alpha$ band) and $6900 \AA$ (B band) were measured in the same way so as to determine the offset between the stellar spectrum and its associated thorium-argon comparison spectrum. The stellar topocentric velocities were then corrected by subtracting from them the telluric line offset in velocity form, and, finally, the heliocentric correction led to heliocentric radial velocities. The McDonald velocities are, thus, absolute velocities and are listed in Table 3.

For the KPNO spectrograms we determined radial velocities with the IRAF cross-correlation program FXCOR (Fitzpatrick 1993), fitting a Gaussian function to the cross-correlation peak. The IAU radial velocity standard star HR 7560 was used as the cross-correlation reference star for the red-wavelength spectrograms. Its velocity of $0.0 \mathrm{~km} \mathrm{~s}^{-1}$ was adopted from Scarfe et al. (1990). Lines in the wavelength region redward of $6445 \AA$ are not particularly suitable for measurement because most features are blends, and there are a number of modest strength water vapor lines. Thus, the radial velocities were determined from lines in the region $6385-6445 \AA$ A. However, this $60 \AA$ portion of the spectrum is so small that a spectrum mismatch, caused by the varying strength of line blends with temperature, between the early-F components of HR 8257, and that of the cross-correlation standard HR 7560, spectral type F8 V (Gray et al. 2001), can significantly alter the measured velocity. Thus, instead of cross-correlating this entire $60 \AA$ wavelength region, only the wavelength regions around the five strongest and leastblended lines, the Fe I lines at 6394, 6412, 6421, and $6431 \AA$ plus the Ca I line at $6439 \AA$, were cross-correlated. The KPNO velocities are also given in Table 3 .

The Coravel radial velocities, also listed in Table 3, were obtained by cross-correlation of the stellar spectra with a template incorporated into the spectrometer. The template was extracted from a spectrum of the K2 III star Arcturus.

\section{ORBIT}

\subsection{Spectroscopic Orbit}

As a prelude to a spectroscopic solution of the velocities from the four sources, we examined the zero points of the four velocity systems by computing independent orbital solutions, four each for the primary and secondary components. Despite the very different methods of velocity reduction, the center-of-mass velocities of the McDonald, KPNO, and CfA velocities agreed to within their $1 \sigma$ uncertainties. In particular, the McDonald and KPNO center-of-mass velocities of the four solutions differed by less than $0.1 \mathrm{~km} \mathrm{~s}^{-1}$. Using velocities from the same three observatories, Tomkin \& Fekel (2006) found similar, excellent zero point agreement in an analysis of the binary $12 \mathrm{Boo}$ (following the 12 Boo report of Boden et al. 2005). The Coravel HPO velocities of HR 8257 were slightly problematic. The center-of-mass velocity of the secondary was in agreement with the results for the other three data sets, but the value for the primary differed by $3 \sigma$. We have chosen not to adjust any of the velocities. 
Table 2

HR 8257 PTI V² Data, Predictions and Residuals from “Joint” Orbit Model

\begin{tabular}{lcccccccccc}
\hline \hline MJD & UT Date & UT Time & $\begin{array}{c}\text { Wavelength } \\
(\mu \mathrm{m})\end{array}$ & $\begin{array}{c}\text { Measured } \\
V^{2}\end{array}$ & $\begin{array}{c}\text { Error in } \\
V^{2}\end{array}$ & $\begin{array}{c}\text { Predicted } \\
V^{2}\end{array}$ & Resid. & \multicolumn{1}{c}{$\begin{array}{c}u \\
(\mathrm{~m})\end{array}$} & $\begin{array}{c}v \\
(\mathrm{~m})\end{array}$ & Phase \\
\hline $51,720.4024$ & 6252000 & $09: 39: 26$ & 2.221 & 0.416 & 0.034 & 0.485 & -0.069 & -6.7263 & -93.9262 & 0.452 \\
$51,720.4097$ & 6252000 & $09: 49: 55$ & 2.223 & 0.487 & 0.024 & 0.518 & -0.030 & -54.7713 & -95.1354 & 0.453 \\
$51,720.4669$ & 6252000 & $11: 12: 15$ & 2.222 & 0.770 & 0.027 & 0.802 & -0.032 & -35.7951 & -102.9270 & 0.457 \\
$51,720.4738$ & 6252000 & $11: 22: 11$ & 2.220 & 0.831 & 0.084 & 0.834 & -0.003 & -33.1381 & -103.6350 & 0.458 \\
$51,720.4878$ & 6252000 & $11: 42: 23$ & 2.221 & 0.955 & 0.070 & 0.895 & 0.061 & -27.5515 & -104.9030 & 0.459 \\
$51,720.4947$ & 6252000 & $11: 52: 22$ & 2.220 & 0.966 & 0.059 & 0.920 & 0.046 & -24.7037 & -105.4430 & 0.459 \\
$51,724.3901$ & 6292000 & $09: 21: 46$ & 2.238 & 0.358 & 0.039 & 0.341 & 0.017 & -57.0752 & -93.6973 & 0.778 \\
$51,724.4043$ & 6292000 & $09: 42: 11$ & 2.246 & 0.326 & 0.047 & 0.331 & -0.004 & -53.2051 & -96.0267 & 0.780 \\
$51,724.4410$ & 6292000 & $10: 35: 02$ & 2.245 & 0.329 & 0.052 & 0.309 & 0.020 & -41.3015 & -101.2140 & 0.783 \\
$51,724.4556$ & 6292000 & $10: 56: 05$ & 2.244 & 0.320 & 0.036 & 0.306 & 0.014 & -35.9115 & -102.8940 & 0.784 \\
$51,724.5093$ & 6292000 & $12: 13: 21$ & 2.245 & 0.377 & 0.042 & 0.324 & 0.052 & -13.8834 & -106.9100 & 0.788 \\
$51,735.3989$ & 7102000 & $09: 34: 24$ & 2.236 & 1.044 & 0.156 & 0.952 & 0.092 & -83.4619 & -22.0325 & 0.680 \\
\hline
\end{tabular}

Notes. Residuals are quoted as data minus model. Visibilities are from PTI synthetic wide-band reductions (Colavita 1999), and effective center-band wavelengths are SNR-weighted across the channels in the PTI spectrometer.

(This table is available in its entirety in a machine-readable form in the online journal. A portion is shown here for guidance regarding its form and content.)

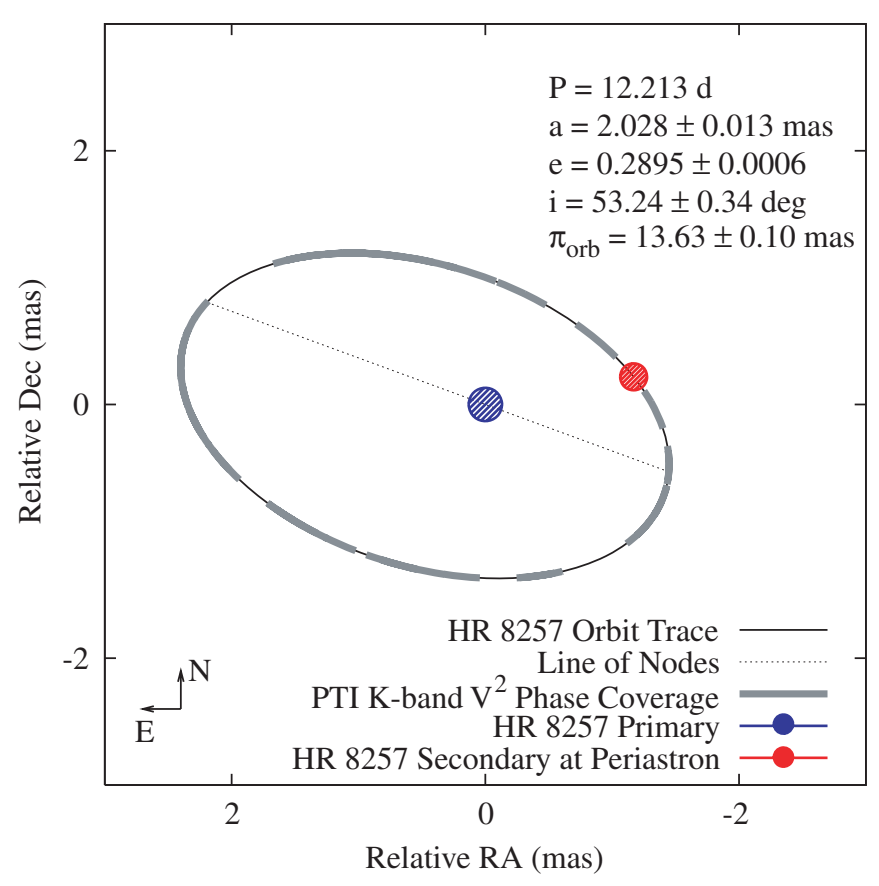

Figure 1. Visual Orbit of HR 8257. The relative visual orbit model of HR 8257 is shown, with the primary and secondary stars rendered at $T$ (periastron). The heavy lines along the relative orbit indicate areas where we have phase coverage in our $K$-band PTI data (they are not separation vector estimates). Essentially all phases of the orbit are well sampled, leading to a reliable orbit determination. Component diameter values are estimated (see Section 5) and rendered to scale.

These separate solutions also showed excellent agreement between the various orbital elements. In particular, the velocity semiamplitudes differed by $1 \sigma$ or less, indicating that there are no significant systematic errors in the data sets that might preclude combining the velocities into a single solution.

The variances of the orbital solutions are inversely proportional to our adopted weights. For the primary we assigned weights of $1.0,1.0,0.04$, and 0.04 to the velocities of McDonald, KPNO, CfA, and HPO, respectively. For the secondary component the weights, corresponding to the same observatory order, became $0.4,0.3,0.04$, and 0.03 . A weight of 1.0 corresponds to a velocity standard deviation of $0.11 \mathrm{~km} \mathrm{~s}^{-1}$ in these radial velocity solutions. In the joint orbital solution discussed below, the standard deviation increased to
$0.15 \mathrm{~km} \mathrm{~s}^{-1}$. For one HPO observation the components were seriously blended, and so that observation was not used in the solutions.

A total of 44 McDonald, KPNO, and CfA velocities was obtained between 2000 and 2006. Combined in a simultaneous solution of the two components, those observations produced an orbital period of $12.21346 \pm 0.00003$. Although low in weight, the HPO velocities, collected between 1982 and 1993, substantially extend the temporal baseline. When included in the orbital solution, they slightly revised the period to $12.21345 \pm$ 0.00001 but do not significantly improve the other orbital elements.

\subsection{Combined Orbit}

As in previous work (e.g., Boden et al. 2005), we have obtained three orbital solutions of HR 8257, one based on the radial velocities, one on the PTI visibilities, and, finally, a joint solution of these two data sets. Methods for binary orbital analysis are discussed in Boden et al. (2000) and not repeated here.

Figure 1 depicts the relative visual orbit of the HR 8257 system, with the primary component rendered at the origin, and the secondary component positioned at periastron. We have indicated the phase coverage of our $V^{2}$ data with heavy lines on the relative orbit. Our data sample essentially all phases of the orbit, leading to a reliable orbit determination. The sizes of the HR 8257 components are estimated (see the discussion in Section 5) and rendered to scale. $V^{2}$ observations are subject to a point-symmetric inversion degeneracy (a scene and its reflection around the phase-center produce the same $V^{2}$ ), so astrometric orbits determined with such data have a modulo $180^{\circ}$ uncertainty in the apparent orientation on the sky and longitude of the ascending node ( $\Omega$; e.g., Boden et al. 2000, 2005). While this degeneracy has no impact on the derived physical parameters, it does mean that there is a $180^{\circ}$ uncertainty in the orbit as rendered in Figure 1.

Figure 2 compares the primary and secondary velocities of the four data sets with the computed radial velocity orbits from our joint interferometric-spectroscopic solution. The specifics are presented in Table 3, where for each observation date we list the velocities of the primary and secondary, the rms errors of the velocities in the data sets relative to the full spectroscopic solution, as well as the observed minus computed velocity 
Table 3

HR 8257 Velocities, Residuals, and Phases

\begin{tabular}{|c|c|c|c|c|c|c|c|c|}
\hline MJD & $\begin{array}{c}\mathrm{V}_{A} \\
\left(\mathrm{~km} \mathrm{~s}^{-1}\right)\end{array}$ & $\begin{array}{c}\mathrm{RMS}_{A} \\
\left(\mathrm{~km} \mathrm{~s}^{-1}\right)\end{array}$ & $\begin{array}{c}\mathrm{V}_{B} \\
\left(\mathrm{~km} \mathrm{~s}^{-1}\right)\end{array}$ & $\begin{array}{c}\mathrm{RMS}_{B} \\
\left(\mathrm{~km} \mathrm{~s}^{-1}\right)\end{array}$ & $\begin{array}{c}(O-C)_{A} \\
\left(\mathrm{~km} \mathrm{~s}^{-1}\right)\end{array}$ & $\begin{array}{c}(O-C)_{B} \\
\left(\mathrm{~km} \mathrm{~s}^{-1}\right)\end{array}$ & $\begin{array}{l}\text { Orbital } \\
\text { Phase }\end{array}$ & $\overline{\text { Source }^{a}}$ \\
\hline $45,174.054$ & -74.6 & 0.8 & -7.5 & 0.9 & -0.1 & -0.1 & 0.457 & HPO \\
\hline $45,231.960$ & -76.5 & 0.8 & -5.1 & 0.9 & 0.7 & -0.7 & 0.198 & HPO \\
\hline $45,271.804$ & -74.6 & 0.8 & -8.1 & 0.9 & -0.5 & -0.3 & 0.460 & HPO \\
\hline $45,564.912$ & -74.0 & 0.8 & -8.4 & 0.9 & 0.3 & -0.7 & 0.459 & HPO \\
\hline $45,619.812$ & 19.7 & 0.8 & -110.7 & 0.9 & 1.5 & 1.4 & 0.954 & HPO \\
\hline $45,625.797$ & -75.0 & 0.8 & -3.8 & 0.9 & 0.9 & 2.0 & 0.444 & HPO \\
\hline $45,917.945$ & -82.3 & 0.8 & 1.6 & 0.9 & 0.1 & 0.1 & 0.364 & HPO \\
\hline $45,919.047$ & -74.6 & 0.8 & -6.8 & 0.9 & 0.2 & 0.3 & 0.455 & HPO \\
\hline $45,920.008$ & -65.5 & 0.8 & -18.8 & 0.9 & -0.9 & -0.2 & 0.533 & HPO \\
\hline $45,923.989$ & 9.8 & 0.8 & -102.3 & 0.9 & 0.8 & -0.5 & 0.859 & HPO \\
\hline $45,924.958$ & 19.8 & 0.8 & -114.1 & 0.9 & 0.2 & -0.4 & 0.938 & HPO \\
\hline $45,925.928$ & -3.9 & 0.8 & -87.9 & 0.9 & 0.0 & -0.8 & 0.018 & HPO \\
\hline $45,928.996$ & -83.4 & 0.8 & 2.8 & 0.9 & 0.4 & -0.3 & 0.269 & HPO \\
\hline $45,930.989$ & -77.3 & 0.8 & -4.4 & 0.9 & -0.2 & 0.1 & 0.432 & HPO \\
\hline $45,941.986$ & -83.4 & 0.8 & 2.9 & 0.9 & 0.4 & -0.2 & 0.333 & $\mathrm{HPO}$ \\
\hline $45,942.952$ & -77.7 & 0.8 & -2.4 & 0.9 & 1.3 & -0.1 & 0.412 & HPO \\
\hline $45,943.933$ & -70.1 & 0.8 & -11.2 & 0.9 & 0.2 & 1.0 & 0.492 & HPO \\
\hline $45,944.957$ & -57.6 & 0.8 & -25.5 & 0.9 & 0.2 & 0.8 & 0.576 & HPO \\
\hline $45,944.961$ & -56.9 & 0.8 & -26.4 & 0.9 & 0.8 & -0.0 & 0.576 & HPO \\
\hline $45,948.934$ & 18.4 & 0.8 & -111.1 & 0.9 & 0.9 & 0.3 & 0.902 & HPO \\
\hline $45,949.933$ & 11.7 & 0.8 & -101.9 & 0.9 & 0.6 & 2.3 & 0.983 & HPO \\
\hline $45,995.834$ & -20.9 & 0.8 & -66.6 & 0.9 & 1.6 & -0.5 & 0.742 & HPO \\
\hline $48,443.075$ & -51.2 & 0.8 & -29.4 & 0.9 & 2.7 & 1.3 & 0.114 & HPO \\
\hline $49,163.054$ & -28.5 & 0.8 & -58.4 & 0.9 & 0.8 & 0.0 & 0.064 & HPO \\
\hline $51,749.312$ & -1.6 & 0.8 & -91.0 & 0.8 & -0.0 & -1.2 & 0.819 & CfA \\
\hline $52,567.088$ & -13.5 & 0.2 & -76.8 & 0.2 & -0.1 & -0.4 & 0.776 & $\mathrm{McD}$ \\
\hline $52,609.080$ & -79.5 & 0.2 & -1.8 & 0.2 & 0.0 & -0.1 & 0.214 & $\mathrm{McD}$ \\
\hline $52,610.061$ & -84.5 & 0.2 & 3.7 & 0.2 & -0.2 & -0.0 & 0.294 & $\mathrm{McD}$ \\
\hline $52,750.470$ & -9.5 & 0.2 & -81.0 & 0.2 & -0.1 & 0.0 & 0.791 & $\mathrm{McD}$ \\
\hline $52,888.283$ & -34.7 & 0.2 & -51.5 & 0.2 & 0.3 & 0.5 & 0.074 & $\mathrm{McD}$ \\
\hline $52,889.311$ & -68.9 & 0.2 & -14.0 & 0.2 & -0.1 & -0.2 & 0.159 & $\mathrm{McD}$ \\
\hline $52,890.251$ & -81.9 & 0.2 & 0.7 & 0.2 & -0.1 & -0.1 & 0.236 & $\mathrm{McD}$ \\
\hline $53,109.467$ & -75.0 & 0.2 & -7.1 & 0.2 & -0.3 & 0.0 & 0.184 & $\mathrm{McD}$ \\
\hline $53,172.394$ & -83.4 & 0.2 & 2.7 & 0.3 & 0.3 & -0.3 & 0.337 & KPNO \\
\hline $53,259.304$ & -75.2 & 0.2 & -6.8 & 0.2 & -0.2 & 0.0 & 0.453 & $\mathrm{McD}$ \\
\hline $53,260.304$ & -64.3 & 0.2 & -18.9 & 0.2 & 0.1 & -0.1 & 0.534 & $\mathrm{McD}$ \\
\hline $53,261.218$ & -52.1 & 0.2 & -33.6 & 0.2 & -0.2 & -0.6 & 0.609 & $\mathrm{McD}$ \\
\hline $53,276.208$ & 3.2 & 0.2 & -95.5 & 0.3 & -0.0 & -0.2 & 0.837 & KPNO \\
\hline $53,321.054$ & -67.2 & 0.8 & -14.4 & 0.8 & 0.9 & 0.2 & 0.508 & CfA \\
\hline $53,334.107$ & -57.4 & 0.2 & -26.3 & 0.2 & 0.2 & 0.2 & 0.577 & $\mathrm{McD}$ \\
\hline $53,336.968$ & -3.5 & 0.8 & -87.6 & 0.8 & 0.2 & -0.1 & 0.811 & CfA \\
\hline $53,355.026$ & -84.5 & 0.8 & 2.8 & 0.8 & -0.2 & -0.9 & 0.290 & CfA \\
\hline $53,475.369$ & -63.8 & 0.8 & -18.9 & 0.8 & 0.6 & -0.1 & 0.143 & CfA \\
\hline $53,533.449$ & 17.0 & 0.2 & -111.0 & 0.3 & -0.1 & -0.0 & 0.899 & KPNO \\
\hline $53,534.443$ & 12.2 & 0.2 & -105.4 & 0.3 & 0.0 & -0.0 & 0.980 & KPNO \\
\hline $53,535.382$ & -25.5 & 0.2 & -62.1 & 0.3 & -0.0 & 0.7 & 0.057 & KPNO \\
\hline $53,547.478$ & -20.5 & 0.8 & -70.2 & 0.8 & -0.4 & -1.3 & 0.047 & CfA \\
\hline $53,630.169$ & -2.0 & 0.8 & -89.8 & 0.8 & -0.1 & -0.3 & 0.818 & CfA \\
\hline $53,637.212$ & -80.5 & 0.2 & -0.6 & 0.3 & -0.1 & 0.1 & 0.394 & KPNO \\
\hline $53,656.238$ & 18.2 & 0.8 & -112.5 & 0.8 & -0.2 & -0.1 & 0.952 & CfA \\
\hline $53,661.150$ & -82.9 & 0.2 & 2.1 & 0.2 & 0.0 & -0.0 & 0.354 & $\mathrm{McD}$ \\
\hline $53,663.143$ & -66.8 & 0.2 & -15.9 & 0.2 & 0.0 & 0.2 & 0.518 & $\mathrm{McD}$ \\
\hline $53,690.130$ & -26.4 & 0.2 & -62.0 & 0.2 & -0.2 & 0.0 & 0.727 & $\mathrm{McD}$ \\
\hline $53,691.138$ & -4.0 & 0.2 & -87.0 & 0.2 & 0.1 & -0.0 & 0.810 & $\mathrm{McD}$ \\
\hline $53,692.061$ & 13.4 & 0.8 & -109.8 & 0.8 & -1.4 & -1.4 & 0.885 & CfA \\
\hline $53,714.101$ & -35.1 & 0.8 & -52.2 & 0.8 & 0.0 & -0.3 & 0.690 & CfA \\
\hline $53,851.511$ & 19.7 & 0.2 & -113.6 & 0.3 & 0.2 & 0.0 & 0.941 & KPNO \\
\hline $53,852.503$ & -5.9 & 0.2 & -84.9 & 0.3 & 0.1 & -0.1 & 0.022 & KPNO \\
\hline $53,855.475$ & -83.3 & 0.2 & 3.0 & 0.3 & 0.4 & 0.0 & 0.265 & KPNO \\
\hline $53,873.485$ & -21.8 & 0.8 & -64.5 & 0.8 & 1.2 & 1.1 & 0.740 & CfA \\
\hline $53,911.462$ & 6.4 & 0.2 & -99.4 & 0.3 & -0.2 & -0.4 & 0.849 & KPNO \\
\hline $53,922.426$ & -20.4 & 0.8 & -68.1 & 0.8 & 0.8 & -0.4 & 0.747 & CfA \\
\hline $54,004.197$ & -76.2 & 0.2 & -5.7 & 0.3 & -0.1 & -0.1 & 0.442 & KPNO \\
\hline $54,020.086$ & -22.6 & 0.8 & -67.0 & 0.8 & -0.4 & -0.4 & 0.743 & CfA \\
\hline $54,046.059$ & 11.6 & 0.2 & -104.5 & 0.2 & 0.1 & 0.1 & 0.870 & $\mathrm{McD}$ \\
\hline
\end{tabular}


Table 3

(Continued)

\begin{tabular}{|c|c|c|c|c|c|c|c|c|}
\hline MJD & $\begin{array}{c}\mathrm{V}_{A} \\
\left(\mathrm{~km} \mathrm{~s}^{-1}\right)\end{array}$ & $\begin{array}{c}\mathrm{RMS}_{A} \\
\left(\mathrm{~km} \mathrm{~s}^{-1}\right)\end{array}$ & $\begin{array}{c}\mathrm{V}_{B} \\
\left(\mathrm{~km} \mathrm{~s}^{-1}\right)\end{array}$ & $\begin{array}{c}\mathrm{RMS}_{B} \\
\left(\mathrm{~km} \mathrm{~s}^{-1}\right)\end{array}$ & $\begin{array}{c}(O-C)_{A} \\
\left(\mathrm{~km} \mathrm{~s}^{-1}\right)\end{array}$ & $\begin{array}{c}(O-C)_{B} \\
\left(\mathrm{~km} \mathrm{~s}^{-1}\right)\end{array}$ & $\begin{array}{c}\text { Orbital } \\
\text { Phase }\end{array}$ & Source ${ }^{\mathrm{a}}$ \\
\hline $54,047.120$ & 17.8 & 0.2 & -111.6 & 0.2 & -0.0 & 0.1 & 0.957 & $\mathrm{McD}$ \\
\hline $54,048.066$ & -12.6 & 0.2 & -77.5 & 0.2 & -0.0 & -0.1 & 0.034 & $\mathrm{McD}$ \\
\hline $54,074.046$ & -69.9 & 0.8 & -13.2 & 0.8 & -0.4 & -0.2 & 0.161 & CfA \\
\hline
\end{tabular}

Note.

${ }^{\mathrm{a}} \mathrm{HPO}=$ Haute-Provence Observatory, CfA = Oak Ridge Observatory or Whipple Observatory, $\mathrm{McD}=$ McDonald Observatory, and KPNO = Kitt Peak National Observatory.

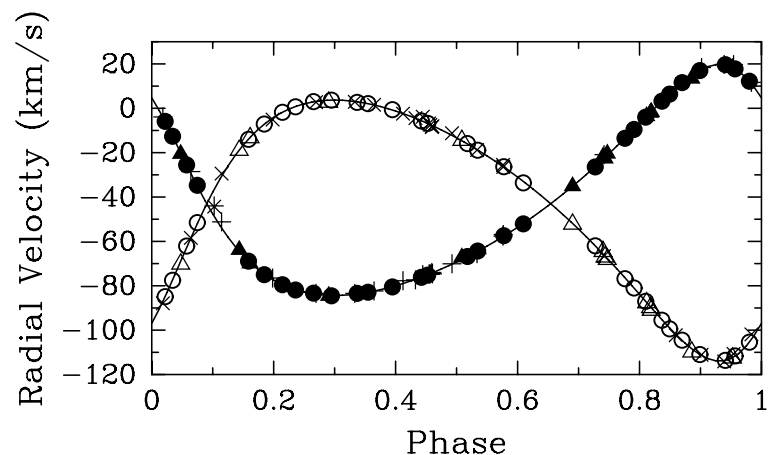

Figure 2. Computed radial-velocity curves of HR 8257 compared with the radial velocities. For component A filled circles represent McDonald Observatory and KPNO velocities, filled triangles are CfA velocities, and pluses are HPO velocities. For component B open circles are McDonald and KPNO velocities, open triangles are CfA velocities, and x symbols correspond to HPO velocities. Zero phase is a time of periastron passage.

residuals from the joint solution. Phases from that solution are given, where zero phase is a time of periastron passage. Finally, the source of each observation is noted.

Table 4 summarizes our orbital models for HR 8257. Included are a velocity-only, a $V^{2}$-only, and a joint orbital solution of both types of data. Solutions with interferometric data have component diameters constrained as noted above. All orbital parameters are listed with total $1 \sigma$ errors, including contributions from statistical (measurement uncertainty) and systematic error sources. In our analysis the dominant forms of systematic error are: (1) uncertainties in the calibrator angular diameters (Table 1); (2) uncertainty in the centerband operating wavelength $\left(\lambda_{0} \approx 2.2 \mu \mathrm{m}\right)$, taken to be $10 \mathrm{~nm}$ ( $\sim 0.5 \%)$; (3) the geometrical uncertainty in our interferometric baseline $(<0.01 \%)$; and $(4)$ uncertainties in ancillary parameters constrained in our orbit fitting procedure (i.e., the angular diameters in all solutions involving interferometry data).

\section{SPECTROSCOPICALLY-DERIVED PROPERTIES}

As noted earlier, to determine the CfA radial velocities requires that the synthetic template spectra be optimized for the two component stars. We obtained $v \sin i$ values of 12 and $7 \mathrm{~km} \mathrm{~s}^{-1}$ for the primary and secondary with estimated errors of 1 and $2 \mathrm{~km} \mathrm{~s}^{-1}$, respectively. The effective temperatures are sensitive to the adopted surface gravity and chemical composition. For an adopted $\log g=4.0$ for both components, we obtained by interpolation temperatures of 7100 and $6700 \mathrm{~K}$ for the primary and secondary with adopted uncertainties of 100 and $150 \mathrm{~K}$, respectively. As a result of the discussion below, solar metallicity was adopted for the system. A secondary to primary luminosity ratio of $0.49 \pm 0.02$ was also determined for the spectral region at $5190 \AA$.
Table 4

Orbital Parameters for HR 8257

\begin{tabular}{lccc}
\hline \hline Parameter & \multicolumn{3}{c}{ Orbital Solutions } \\
\cline { 2 - 4 } & RV-Only & $V^{2}$-Only & Joint \\
\hline Period (days) & 12.213448 & 12.21345 & 12.213446 \\
& $\pm 1.0 \times 10^{-5}$ & $\pm 1.0 \times 10^{-4}$ & $\pm 1.0 \times 10^{-5}$ \\
$T$ (MJD) & $51,372.9036$ & $51,372.951$ & $51,372.9064$ \\
& \pm 0.0041 & \pm 0.021 & \pm 0.0040 \\
& 0.28974 & 0.2924 & 0.28953 \\
$K_{A}\left(\mathrm{~km} \mathrm{~s}^{-1}\right)$ & \pm 0.00059 & \pm 0.0034 & \pm 0.00057 \\
$K_{B}\left(\mathrm{~km} \mathrm{~s}^{-1}\right)$ & $52.059 \pm 0.038$ & $\ldots$ & $52.054 \pm 0.038$ \\
$\gamma\left(\mathrm{km} \mathrm{s}^{-1}\right)$ & $58.835 \pm 0.061$ & $\ldots$ & $58.829 \pm 0.061$ \\
$\omega_{A}(\mathrm{deg})$ & $-43.003 \pm 0.023$ & $\ldots$ & $-43.001 \pm 0.023$ \\
$\Omega(\mathrm{deg})$ & $44.71 \pm 0.13$ & $46.13 \pm 0.81$ & $44.79 \pm 0.12$ \\
$i(\mathrm{deg})$ & $\ldots$ & $69.54 \pm 0.51$ & $69.85 \pm 0.19$ \\
$a(\mathrm{mas})$ & $\ldots$ & $53.46 \pm 0.40$ & $53.23 \pm 0.34$ \\
$\Delta K_{\mathrm{CIT}}\left(\mathrm{mag}^{2}\right)$ & $\ldots$ & $2.031 \pm 0.014$ & $2.028 \pm 0.013$ \\
$\chi^{2} / \mathrm{dof}$ & $\ldots$ & $0.593 \pm 0.029$ & $0.601 \pm 0.027$ \\
$\left|R_{V^{2}}\right| / \sigma_{V^{2}}$ & $\ldots$ & 1.063 & 1.085 \\
$\left|R_{R V}\right| / \sigma_{R V}\left(\mathrm{~km} \mathrm{~s}^{-1}\right)$ & $\ldots$ & $0.0595 / 0.1068$ & $0.0595 / 0.1066$ \\
\hline
\end{tabular}

Notes. Summarized here are the orbital parameters for the HR 8257 system as determined in the present work. We give three separate fits to our data: RV-only, $V^{2}$-only, and both data sets jointly ("Joint"). $\Omega$ is reported in a position angle convention.

Strassmeier \& Fekel (1990) identified several luminositysensitive and temperature-sensitive line ratios in the 6430$6465 \AA$ region. Those critical line ratios and the general appearance of the spectrum were employed as spectral-type criteria. However, for stars that are hotter than about early-G, the line ratios in the $6430 \AA$ region have little sensitivity to luminosity, so only the spectral class of an A or F star can be determined. The luminosity class is determined by computing the absolute visual magnitude with our orbital parallax and comparing that magnitude to evolutionary tracks or a table of canonical values for giants and dwarfs.

The spectrum of HR 8257 was compared with those of slowly rotating late-A and early- to mid-F stars. These reference stars, identified mostly from the lists of Abt \& Morrell (1995) and Fekel (1997), were observed at the KPNO with the same telescope, spectrograph, and detector as our spectra of HR 8257. With a computer program developed by Huenemoerder \& Barden (1984) and Barden (1985), various combinations of reference-star spectra were rotationally broadened, shifted in radial velocity, appropriately weighted, and added together in an attempt to reproduce the spectrum of HR 8257 in the $6430 \AA$ region. Reference stars with late-A or $\mathrm{F}$ spectral classes and $[\mathrm{Fe} / \mathrm{H}]=-0.3$ do not provide a good fit to the spectrum. Instead, the best fit was found with reference stars that have essentially solar iron abundances and spectral classes of F0 and F2 for 
HR 8257--F0IV SED Model
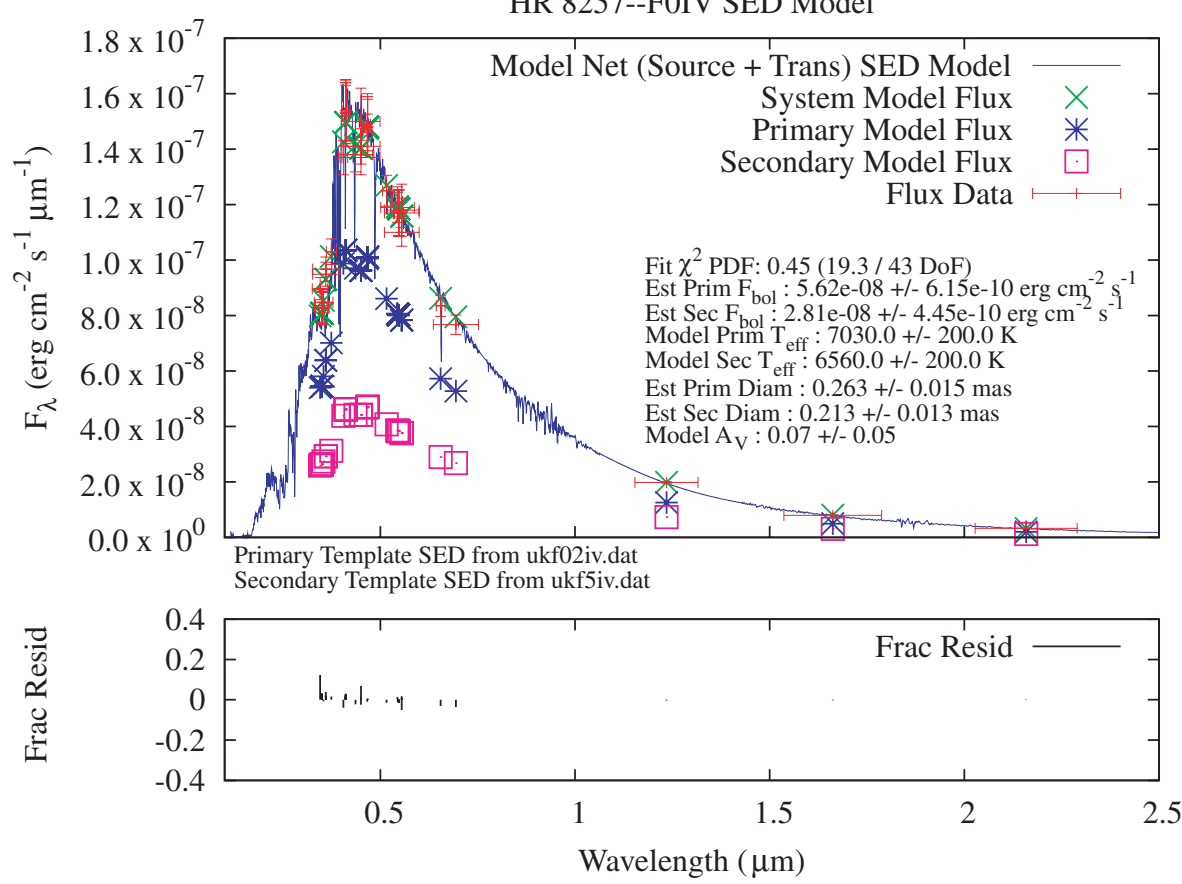

HR 8257--F0IV Binary Source Ratios

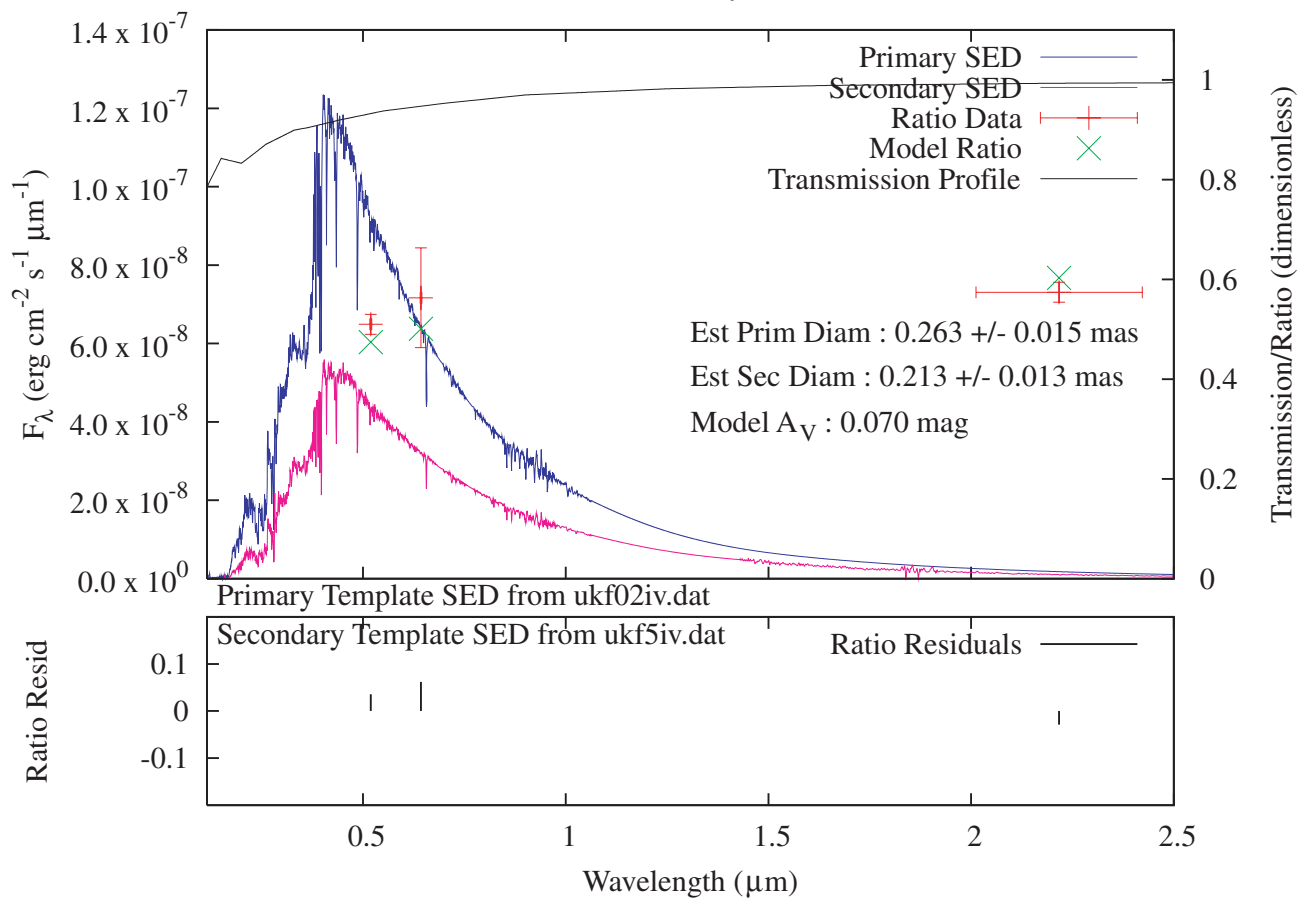

Figure 3. SED model of HR 8257. The SED templates of Pickles (1998) have been simultaneously fitted to archival photometry (top) and flux ratio estimates (bottom) from our visibility and spectroscopic measurements (Table 4).

the primary and secondary, respectively. Thus, we estimate that the abundances of the components of HR 8257 are also close to solar with $[\mathrm{Fe} / \mathrm{H}] \sim 0.0 \pm 0.2$. In particular, the iron and calcium line strengths of both components are reproduced by stars with normal spectra, so neither component of this close binary is an Am star. This finding is consistent with classifications of the combined spectrum by expert classifiers (Abt \& Bidelman 1969; Cowley \& Fraquelli 1974; Abt \& Morrell 1995). The effective temperatures of the components derived from the CfA spectra imply spectral classes of A9 and F2, according to
Table B1 of Gray (1992), and so are in good agreement with our classifications.

The continuum intensity ratio at $6430 \AA$ of our best reference star combination, fitted to the spectrum of HR 8257, is 0.53 with an estimated uncertainty of 0.04 . The uncertainty is based on how well the various reference star spectra account for the binary spectrum plus uncertainty in the abundances of the reference stars. Because the spectral classes of the two stars are very similar, we adopt the continuum intensity ratio as the luminosity ratio at $6430 \AA$, a wavelength that is about 0.6 of the way between 

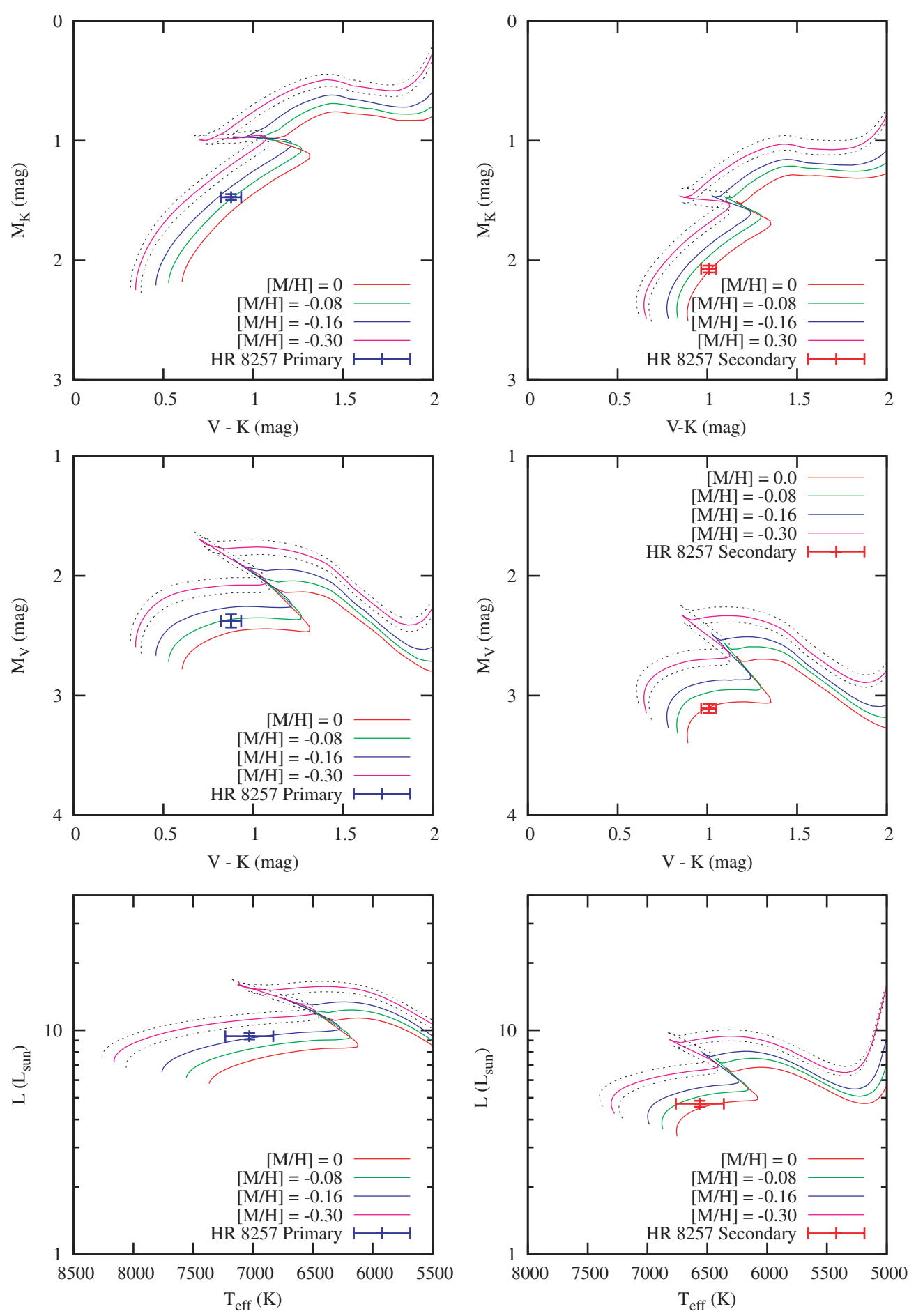

Figure 4. Positions of the binary components (primary: left column; secondary: right column) of HR 8257 are plotted in each of the color-magnitude and theoretical $\mathrm{H}-\mathrm{R}$ diagrams. Computed $\mathrm{Y}^{2}$ evolutionary tracks, corresponding to the component dynamical masses for metal abundances of $[\mathrm{M} / \mathrm{H}]=0.0,-0.08,-0.16,-0.30$, are shown for comparison. $1 \sigma$ variations in the tracks as a function of the dynamical mass errors (Table 5) are shown on the $[\mathrm{M} / \mathrm{H}]=-0.30$ tracks. There is good agreement between the $\mathrm{Y}^{2}$ tracks and the observed HR 8257 component properties for a plausible abundance range of $-0.16<[\mathrm{M} / \mathrm{H}]<0$. Both stars are on the main sequence and have not yet entered the subgiant phase.

the center of the Johnson $V$ and $R$ bandpasses. This luminosity ratio corresponds to a magnitude difference of 0.69 with an estimated uncertainty of 0.1 .

With the procedure of Fekel (1997), we determined projected rotational velocities for the components of HR 8257 from $10 \mathrm{KPNO}$ red-wavelength spectra. For each spectrum the fullwidths at half-maximum of several lines in the $6430 \AA$ region were measured and the results averaged for each component. The instrumental broadening was removed, and the calibration polynomial of Fekel (1997) was used to convert the resulting broadening in angstroms into a total line broadening in $\mathrm{km} \mathrm{s}^{-1}$. From Fekel (1997) we assumed a macroturbulence of $5 \mathrm{~km} \mathrm{~s}^{-1}$ for early-F stars. The resulting $v$ sin $i$ values are 10 and $7 \mathrm{~km} \mathrm{~s}^{-1}$ for the primary and secondary, respectively. Given 
Table 5

Physical Parameters for HR 8257

\begin{tabular}{|c|c|c|}
\hline $\begin{array}{l}\text { Physical } \\
\text { Parameter }\end{array}$ & $\begin{array}{l}\text { Primary (A) } \\
\text { Component }\end{array}$ & $\begin{array}{l}\text { Secondary (B) } \\
\text { Component }\end{array}$ \\
\hline System Distance (pc) & \multirow{2}{*}{\multicolumn{2}{|c|}{$\begin{array}{c}73.36 \pm 0.51 \\
13.632 \pm 0.095\end{array}$}} \\
\hline$\pi_{\text {orb }}$ (mas) & & \\
\hline Spectral Type & F0 dwarf & F2 dwarf \\
\hline Mass $\left(\mathrm{M}_{\odot}\right)$ & $1.561 \pm 0.021$ & $1.385 \pm 0.019$ \\
\hline$a\left(10^{-2} \mathrm{AU}\right)$ & $6.982 \pm 0.033$ & $7.8912 \pm 0.0082$ \\
\hline Bolometric Flux $\left(10^{-9} \mathrm{erg} \mathrm{cm}^{-2} \mathrm{~s}^{-1}\right)$ & $56.21 \pm 0.62$ & $28.09 \pm 0.44$ \\
\hline$T_{\text {eff }}(\mathrm{K})$ & $7030 \pm 200$ & $6560 \pm 200$ \\
\hline Model Diameter (mas) & $0.263 \pm 0.015$ & $0.213 \pm 0.013$ \\
\hline Luminosity $\left(L_{\odot}\right)$ & $9.41 \pm 0.30$ & $4.70 \pm 0.15$ \\
\hline Radius $\left(R_{\odot}\right)$ & $2.07 \pm 0.12$ & $1.682 \pm 0.10$ \\
\hline $\log g$ & $3.997 \pm 0.048$ & $4.132 \pm 0.052$ \\
\hline$M_{K-\mathrm{CIT}}(\mathrm{mag})$ & $1.472 \pm 0.025$ & $2.073 \pm 0.029$ \\
\hline$M_{V}(\mathrm{mag})$ & $2.377 \pm 0.054$ & $3.108 \pm 0.037$ \\
\hline$V-K^{\mathrm{a}}(\mathrm{mag})$ & $0.878 \pm 0.056$ & $1.008 \pm 0.042$ \\
\hline
\end{tabular}

Notes. Summarized here are the physical parameters for the HR 8257 system as derived primarily from the joint solution orbital parameters in Table 4.

${ }^{\text {a }}$ Color is in the Johnson system.

an estimated uncertainty of $1 \mathrm{~km} \mathrm{~s}^{-1}$ for both values, these rotational velocities are in reasonable accord with those found from the CfA spectra. We average the different determinations and obtain 11 and $7 \mathrm{~km} \mathrm{~s}^{-1}$ for the primary and secondary, respectively.

\section{PHYSICAL PARAMETERS}

The parameters from the joint orbital solution (Table 4) enable us to compute a number of the physical properties of the HR 8257 system and its components, which are summarized in Table 5. Of particular interest is the direct determination of the binary component masses, $1.561 \pm 0.021$ and $1.385 \pm 0.019 M_{\odot}$ for components $\mathrm{A}$ and $\mathrm{B}$, respectively. These uncertainties are 6-7 times larger than those of the minimum masses, and so it is the uncertainty of our orbital inclination, through the $\sin ^{3} i$ factor, that dominates the mass uncertainties. Nevertheless, our masses are well determined, having uncertainties of $\sim 1.4 \%$.

The Hipparcos parallax of HR 8257 is $13.90 \pm 0.73$ mas (Perryman et al. 1997), which corresponds to a distance of $71.9 \pm 3.8 \mathrm{pc}$. The linear and angular separations determined from our joint model produce an orbital parallax of $13.632 \pm$ 0.095 mas, which differs from the Hipparcos result by just $2 \%$ and has a significantly smaller uncertainty.

To determine additional basic properties of the system and its components, we resort to spectral energy distribution (SED) modeling. As input, we have used archival photometry and the estimated component flux ratios in the spectroscopic and visibility data (Table 4). We have fit these data with custom two-component SED modeling code and template SEDs from Pickles (1998). Results from this SED modeling are shown in Figure 3 and listed in Table 5.

The effective temperatures of the best match SEDs for the two components are within $1 \sigma-2 \sigma$ of our other estimates, which were discussed in Section 4. We also compared the SED temperatures for the two components with temperatures obtained from our computed individual $V-K$ colors (Table 5) and the infrared flux method (IRFM) calibration of Alonso et al. (1996) That calibration results in effective temperatures of $6846 \mathrm{~K}$ and $6590 \mathrm{~K}$ for the primary and secondary, respectively. The temperature for the secondary is essentially identical to our SED result. The value for the primary, however, is nearly
$200 \mathrm{~K}$ lower than the SED temperature, but it is still within the SED uncertainty. Adopting an average of the SED and IRFM temperatures for each component slightly enhances the agreement with evolutionary tracks, which are discussed in Section 6.1. However, given that minimal improvement, we have chosen to adopt the SED temperatures. To reduce significantly the temperature uncertainties requires measuring the angular diameters of the components. However, at the distance of HR 8257 neither component is significantly resolved by the PTI $H$ - or $K$-band fringe spacings.

Integrating the model component SEDs, we estimate bolometric fluxes of $(56.21 \pm 0.62) \times 10^{-9} \mathrm{erg} \mathrm{cm}^{-2} \mathrm{~s}^{-1}$ and $(28.09 \pm 0.44) \times 10^{-9} \mathrm{erg} \mathrm{cm}^{-2} \mathrm{~s}^{-1}$ for the primary and secondary components, respectively. When we combine these results with our component model effective temperatures (Table 5), we estimate apparent angular diameters $0.263 \pm$ 0.015 and $0.213 \pm 0.013$ mas for components $\mathrm{A}$ and $\mathrm{B}$, respectively. At the distance of HR 8257 (Table 5) the bolometric fluxes imply luminosities of $9.41 \pm 0.30$ and $4.70 \pm 0.15 L_{\odot}$ and physical radii of $2.07 \pm 0.12$ and $1.682 \pm 0.079 R_{\odot}$ for the primary and secondary stars, respectively.

\section{EVOLUTIONARY STATE}

\subsection{Comparison with Stellar Evolution Models}

With our estimates of the component masses, absolute magnitudes, color indices, and effective temperatures derived from our measurements and orbital solution (Table 5), we examine the physical state of HR 8257 components in the context of recent stellar evolution models from the Yonsei-Yale $\left(\mathrm{Y}^{2}\right)$ collaboration (Yi et al. 2001; Demarque et al. 2004).

As is the case for most binaries, there is no spectroscopic abundance analysis of the components of HR 8257. The Stromgren and $\mathrm{H} \beta$ photometry of Jordi et al. (1996), combined with the relation from Figure 17 of Crawford (1975), results in $[\mathrm{Fe} / \mathrm{H}]=-0.1$ for the combined system, while Nordström et al. (2004) obtained $[\mathrm{Fe} / \mathrm{H}]=-0.16$ from their photometric calibration. We caution that these estimates are based on the combined light of the two stars with the secondary being about $400 \mathrm{~K}$ cooler than the primary. Nevertheless, taken at face value, the abundances indicate that the system is slightly metal poor compared to the Sun. Our analysis (Section 4) of the 6430 A region indicates that the components have near-solar abundances, and the slightly metal poor results derived from photometry are consistent with our result and estimated uncertainty.

In Figure 4 the inferred properties of the HR 8257 components are compared with $\mathrm{Y}^{2}$ evolutionary tracks, computed for a range of metal abundances, in color-magnitude and theoretical H-R diagrams. The left column of Figure 4 compares the observed properties of the primary star with evolutionary tracks computed for the primary dynamical mass and $[\mathrm{M} / \mathrm{H}]$ abundances of 0.0 , $-0.08,-0.16$, and -0.30 . (The $[\mathrm{M} / \mathrm{H}]=-0.30$ track shows the $1 \sigma$ variations with the component dynamical mass errors; Table 5.) Similarly, the right column gives the corresponding comparisons for the HR 8257 secondary. Taken at face value, the comparisons suggest that the primary is slightly more metal poor than the secondary, with the greatest abundance discrepancy occurring in the plot of the luminosity versus effective temperature. However, the observed component properties are only approximately $1 \sigma$ away from a common abundance of $[\mathrm{M} / \mathrm{H}]=-0.08$. Given that this short-period binary consists of two detached dwarf stars, identical abundances would be expected. The diagrams also show that both components 

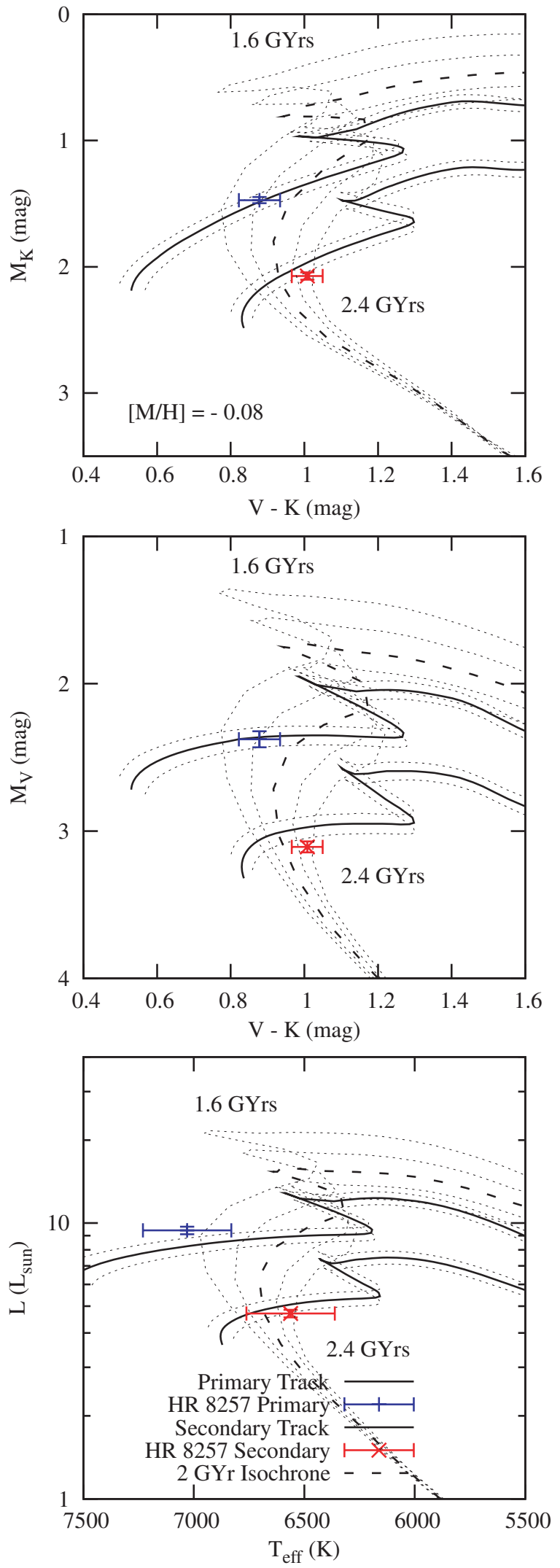

Figure 5. HR 8257 components are shown in observational and theoretical $\mathrm{H}-\mathrm{R}$ diagrams along with $\mathrm{Y}^{2}$ evolutionary tracks at the dynamical masses (with uncertainties) and isochrones all computed at an abundance of $[\mathrm{M} / \mathrm{H}]=-0.08$. Isochrones are given at a range of ages around 2.0 Gyr in $200 \mathrm{Myr}$ steps. These model comparisons indicate an age of $2.0 \pm 0.3 \mathrm{Gyr}$. have evolved from the zero-age main sequence but neither has reached the subgiant stage, so both stars are still dwarfs.

To estimate the age of the system, the components of HR 8257 are compared with the $\mathrm{Y}^{2}$ evolutionary tracks at the dynamical masses (with uncertainties) and isochrones for our adopted abundance of $[\mathrm{M} / \mathrm{H}]=-0.08$ in observational and theoretical $\mathrm{H}-\mathrm{R}$ diagrams (Figure 5). The isochrones are given at a range of ages around 2.0 Gyr in steps of $200 \mathrm{Myr}$. From these model comparisons we adopt an age of $2.0 \pm 0.3 \mathrm{Gyr}$.

\subsection{Circularization and Synchronization}

It is well known (e.g., Tassoul \& Tassoul 1996) that tidal interactions affect the rotational and orbital characteristics of close binaries, causing them to tend toward a state in which the rotational axes of the components are parallel to the orbital axis and their rotational velocities are synchronized with the orbital period. In addition, tidal dissipation of energy causes a binary to circularize its orbit. Indeed, observational results indicate that many binaries partially or fully accomplish these feats (e.g., Duquennoy \& Mayor 1991).

While A stars have radiative outer atmospheres, early-F stars begin to have very thin convective outer atmospheres (Böhm-Vitense 1992), and the convection zone of a star increases in depth as the effective surface temperature decreases. Thus, the primary and secondary components of HR 8257, which have early $\mathrm{F}$ spectral classes, are at the dividing line and have radiative, or at most, extremely thin convective outer envelopes.

To account for tidal friction in binary stars with radiative envelopes, Zahn (1977) investigated the effects of radiative damping on synchronization and circularization, while for components with convective envelopes Zahn (1977, 1989) examined the effects of the equilibrium tide. Meanwhile, Tassoul $(1987,1988)$ explored the theory that binary synchronization and circularization occur because of distortions that cause large scale hydrodynamic currents. In all cases the theories predict that synchronization will occur before circularization.

The orbit of HR 8257 is not circular but instead has a substantial eccentricity of 0.2895 . Thus, the rotational angular velocity of the components will tend to synchronize with that of the orbital motion at periastron, a condition called pseudosynchronous rotation (Hut 1981). With Equation (42) of Hut (1981) we calculated a pseudosynchronous period of 8.1 days.

Combining our radii from Table 5 with the computed pseudosynchronous period results in equatorial rotational velocities of 13.1 and $10.6 \mathrm{~km} \mathrm{~s}^{-1}$ for components $\mathrm{A}$ and $\mathrm{B}$, respectively. Adopting our orbital inclination as the rotational inclination converts our observed $v \sin i$ values of 11 and $7 \mathrm{~km} \mathrm{~s}^{-1}$ to 13.7 and $8.7 \mathrm{~km} \mathrm{~s}^{-1}$ for the primary and secondary components, respectively. Given the $1 \mathrm{~km} \mathrm{~s}^{-1}$ uncertainties of our projected rotational velocities, we find that the primary is rotating pseudosynchronously. The secondary, on the other hand, appears to be close to but has not quite achieved pseudosynchronous rotation.

We thank D. Queloz for contributing the Coravel radial velocities. We are also grateful to P. Berlind, J. Caruso, G. Esquerdo, and R. P. Stefanik for their help with the CFA observations, and to R. J. Davis for maintaining the CFA echelle database. PTI science operations are conducted through the efforts of the PTI Collaboration; we acknowledge the 
invaluable contributions of our PTI colleagues, and particularly thank K. Rykoski for his professional operation of PTI. This research at Tennessee State University has been supported in part by NASA grant NCC5-511 and NSF grant HRD-9706268. G. Torres acknowledges partial support from NSF grant AST0708229 and NASA's MASSIF SIM Key Project (BLF57-04).

\section{REFERENCES}

Abt, H. A., \& Bidelman, W. P. 1969, ApJ, 158, 1091

Abt, H. A., \& Morrell, N. I. 1995, ApJS, 99, 135

Alonso, A., Arribas, S., \& Martínez-Roger, C. 1996, A\&A, 313, 873

Baranne, A., Mayor, M., \& Poncet, J. L. 1979, Vistas Astron., 23, 279

Barden, S. C. 1985, ApJ, 295, 162

Boden, A., Colavita, M., van Belle, G., \& Shao, M. 1998, Proc. SPIE, 3350 872

Boden, A., Creech-Eakman, M., \& Queloz, D. 2000, ApJ, 536, 880

Boden, A. F., Torres, G., \& Hummel, C. A. 2005, ApJ, 627, 464

Boden, A. F., Torres, G., \& Latham, D. W. 2006, ApJ, 644, 1193

Böhm-Vitense, E. 1992, Introduction to Stellar Astrophysics 3 (Cambridge: Cambridge Univ. Press), 81

Colavita, M. M. 1999, PASP, 111, 111

Colavita, M. M., et al. 1999, ApJ, 510, 505

Cowley, A. P., \& Fraquelli, D. 1974, PASP, 86, 70

Crawford, D. L. 1975, AJ, 80, 955

Demarque, P., Woo, J.-H., Kin, Y.-C., \& Yi, S. K. 2004, ApJS, 155, 667

Duquennoy, A., \& Mayor, M. 1991, A\&A, 248, 485

Fekel, F. C. 1997, PASP, 109, 514

Fekel, F. C., \& Tomkin, J. 2004, AN, 325, 649

Fitzpatrick, M. J. 1993, in ASP Conf. Ser. 52, Astronomical Data Analysis Software and Systems II, ed. R. J. Hanisch, R. V. J. Brissenden, \& J. Barnes (San Francisco, CA: ASP), 472

Gray, D. F. 1992, The Observation and Analysis of Stellar Photospheres (Cambridge: Cambridge Univ. Press)

Gray, R. O., Napier, M. G., \& Winkler, L. I. 2001, AJ, 121, 2148
Harper, W. E. 1925, Publ. Dom. Astrophys. Obs., 3, 225

Harper, W. E. 1935, Publ. Dom. Astrophys. Obs., 6, 207

Huenemoerder, D. P., \& Barden, S. C. 1984, BAAS, 16, 510

Hummel, C. A., Mozurkewich, D., Armstrong, J. T., Hajian, A. R., Elias, N. M., \& Hutter, D. J. 1998, AJ, 116, 2536

Hut, P. 1981, A\&A, 99, 126

Jordi, C., Figueras, F., Torra, J., \& Asiain, R. 1996, A\&AS, 115, 401

Latham, D. W. 1992, in ASP Conf. Ser. 32, IAU Colloquium 135, Complementary Approaches to Double and Multiple Star Research, ed. H. A. McAlister \& W. I. Hartkopf (San Francisco, CA: ASP), 110

McAlister, H. A., Hartkopf, W. I., Hutter, D. J., Shara, M. M., \& Franz, O. G. 1987, AJ, 92, 183

McAlister, H. A., Mason, B. D., Hartkopf, W. I., \& Shara, M. M. 1993, AJ, 106, 1639

McCarthy, J. A., Sandiford, B. A., Boyd, D., \& Booth, J. 1993, PASP, 105, 881

Moore, C. E., Minnaert, M. G. J., \& Houtgast, J. 1966, The Solar Spectrum $2935 \AA$ to $8770 \AA$, NBS monograph 61 (Washington, D.C.: U.S. Government Printing Office)

Nordström, B., et al. 2004, A\&A, 418, 989

Perryman, M. A. C., et al. 1997, The Hipparcos and Tycho Catalogues (ESA SP-1200; Noordwijk: ESA)

Petrie, R. M. 1950, Publ. Dom. Astrophys. Obs., 8, 319

Pickles, A. 1998, PASP, 110, 863

Scarfe, C. D., Batten, A. H., \& Fletcher, J. M. 1990, Publ. Dom. Astrophys. Obs., 18, 21

Strassmeier, K. G., \& Fekel, F. C. 1990, A\&A, 230, 389

Tassoul, J.-L. 1987, ApJ, 322, 856

Tassoul, J.-L. 1988, ApJ, 324, L71

Tassoul, J.-L., \& Tassoul, M. 1996, Fundam. Cosm. Phys., 16, 377

Tomkin, J., \& Fekel, F. C. 2006, AJ, 131, 2652

Torres, G., Boden, A., Latham, D., Pan, M, \& Stefanik, R. 2002, AJ, 124, 1716

Yi, S., Demarque, P., Kim, Y.-C., Lee, Y.-W., Ree, C. H., Lejeune, T., \& Barnes, S. 2001, ApJS, 136, 417

Zahn, J.-P. 1977, A\&A, 57, 383

Zahn, J.-P. 1989, A\&A, 220, 112

Zucker, S., \& Mazeh, T. 1994, ApJ, 420, 806 